\title{
Tracking of maternal self-efficacy for limiting young children's television viewing and associations with children's television viewing time: a longitudinal analysis over 15-months
}

\author{
Jill A Hnatiuk, Jo Salmon, Karen J. Campbell, Nicola D. Ridgers and Kylie D. Hesketh
}

\begin{abstract}
Background: Mothers' self-efficacy for limiting their children's television viewing is an important correlate of this behaviour in young children. However, no studies have examined how maternal self-efficacy changes over time, which is potentially important during periods of rapid child development. This study examined tracking of maternal self-efficacy for limiting young children's television viewing over 15-months and associations with children's television viewing time.
\end{abstract}

Methods: In 2008 and 2010, mothers ( $n=404$ ) from the Melbourne InFANT Program self-reported their self-efficacy for limiting their child's television viewing at 4- and 19-months of age. Tertiles of self-efficacy were created at each time and categorised into: persistently high, persistently low, increasing or decreasing self-efficacy. Weighted kappa and multinomial logistic regression examined tracking and demographic and behavioural predictors of change in self-efficacy. A linear regression model examined associations between tracking categories and children's television viewing time.

Results: Tracking of maternal self-efficacy for limiting children's television viewing was low (kappa $=0.23, p<0.001$ ). Mothers who had persistently high or increasing self-efficacy had children with lower television viewing time at 19-months ( $\beta=-35.5 ; 95 \% \mathrm{Cl}=-54.4,-16.6$ and $\beta=37.0 ; 95 \% \mathrm{Cl}=-54.4,-19.7$, respectively). Mothers of children with difficult temperaments were less likely to have persistently high self-efficacy. Mothers who met adult physical activity guidelines had 2.5 greater odds of increasing self-efficacy.

Conclusions: Interventions to increase and maintain maternal self-efficacy for limiting children's television viewing time may result in lower rates of this behaviour amongst toddlers. Maternal and child characteristics may need to be considered when tailoring interventions.

Keywords: Television, Infant, Toddler, Maternal behaviour, Tracking

\section{Background}

Limiting the time that children spend watching television is recommended for minimizing negative health outcomes such as increased adiposity and poorer psychosocial health and cognitive development $[1,2]$. As such, current guidelines recommend that children under two years of age do not engage in any television viewing $[3,4]$. However,

\footnotetext{
* Correspondence: j.hnatiuk@uws.edu.au

Centre for Physical Activity and Nutrition Research, Deakin University, 221 Burwood Highway, Burwood, VIC 3125, Australia
}

by 3 months of age, $40 \%$ of American children watch television, and this proportion increases to approximately $90 \%$ by 24 months of age [5]. As mothers play an important gatekeeper role in their child's health behaviours [6], understanding how mothers perceive their ability to facilitate or limit these behaviours and how this changes over time is likely to provide valuable insights into the development of effective interventions to promote children's health behaviours.

Mothers' self-efficacy to support or limit her children's health behaviours is likely to be an important 
influence [7-9]. Self-efficacy refers to an individual's confidence to engage in a particular behaviour under certain conditions [10]; for example, a mothers' confidence in her ability to limit her child's television viewing time when the child is fussing. Cross-sectional evidence suggests that maternal self-efficacy for limiting children's television viewing is associated with less television viewing time $[7,8]$ as well as a lower likelihood of exceeding screen time recommendations [11]. However there is also evidence that mothers' self-efficacy may decrease over time $[8,11]$. Mothers of younger children have reported higher self-efficacy [8] and higher optimism [12] about their ability to influence their child's television viewing behaviours than mothers of older children suggesting that as children get older this may result in decreased maternal self-efficacy to limit their television viewing [8]. However, to date no longitudinal research has confirmed this.

The concept of tracking refers to an individual's rank order position relative to others in the same cohort over a period of time [13]. In this context, it would signify how mothers' self-efficacy is maintained in rank order relative to her peers. If high tracking exists, this can be useful for predicting which mothers may need to be provided with greater support from early in their child's life as this low self-efficacy will tend to persist as their child grows up. However, should maternal self-efficacy change over time, an examination into patterns of movement (e.g. increasing or decreasing self-efficacy) may be necessary.

By identifying characteristics of mothers who are at risk of having low self-efficacy or decreasing self-efficacy over time it may be possible to identify mothers who would benefit from having greater support and strategies available to increase and/or maintain their self-efficacy. Maternal psychological states (e.g. anxiety, depression, education level) and child characteristics (e.g. temperament) are known to influence maternal self-efficacy generally $[14,15]$, yet few studies have examined self-efficacy in relation to children's television viewing specifically (domainspecific self-efficacy). The only variable identified that has been associated with maternal self-efficacy for limiting television viewing is maternal self-reported physical activity level [16]. However, previous research has found that both maternal and child demographic characteristics (e.g. lower maternal education, older child age) and maternal and child behaviours (e.g. higher maternal television viewing time and negative child temperament) are positively associated with children's television viewing time [17-19]. It is therefore possible that these factors also explain changes in mothers' self-efficacy for limiting television viewing behaviours across the early childhood period.

Therefore, the purpose of this study was to examine the tracking of maternal self-efficacy for limiting young children's television viewing and associations with children's television viewing time. A secondary aim was to identify demographic (mothers' education level, child age) and behavioural (mothers' television viewing time and physical activity level and child temperament) predictors of mothers who maintained, increased or decreased their self-efficacy for liming television viewing over the first two years of their children's lives.

\section{Methods}

\section{Participants and procedures}

The participants for this study were drawn from the Melbourne InFANT Program, a cluster-randomized controlled trial promoting obesity-protective behaviours in young children. The trial was delivered to 542 first-time mothers from when their children were 4-months of age and ended when their child was 19-months of age. No intervention effects were observed for maternal self-efficacy for limiting television viewing, therefore participants from both the intervention and control groups were included for the purpose of this study. Self-report questionnaires were completed by mothers at baseline in 2008 (T1- child aged 4-months) and program conclusion in 2010 (T2- child aged 19-months). The study was approved by the Deakin University Human Research Ethics Committee and the Victorian Government's Office for Children. All participants provided informed consent prior to taking part in the study.

\section{Measures \\ Demographic and behavioural predictors at $T 1$}

Mothers reported their highest level of education (categorized as 'low' [secondary school or lower], 'medium' [trade/certificate qualification], or 'high' [university degree or higher]), their age, and the age and sex of their child.

Child temperament was reported by mothers using a single item from the Australian Temperament Project [20]. Mothers rated on a 5-point Likert-type scale the difficulty of their child relative to other children (much easier than average $=0$; much more difficult than average $=4$ ). As few mothers reported their child was 'much more difficult than average', this response category was combined with the 'more difficult than average' response category for use in the analyses.

Mothers' physical activity was assessed using the Active Australia Survey, a reliable and valid measure of self-reported leisure-time physical activity [21, 22]. Following instrument protocols [21], total physical activity (mins/week) was determined by summing the time spent walking ( $>10$ minutes), the time spent in moderateintensity physical activity (MPA) and twice the time spent in vigorous-intensity physical activity (VPA) in the past week. Minutes spent in any given activity intensity were truncated at 840 minutes/week and time spent in MPA and VPA combined (MVPA) was truncated at 1680 minutes/week [21]. Based on the average time 
spent in MVPA per week mothers were either categorized as meeting ( $\geq 150 \mathrm{mins} /$ week) or not meeting ( $<150$ mins/week) physical activity recommendations.

Maternal television viewing time (mins/week) was assessed for weekdays and weekend days by the questions, "On a usual weekday (weekend day), about how many hours do you usually spend sitting down and watching television or videos/DVDs?", previously shown to be valid and reliable [23]. Television viewing time (mins/day) was calculated using a weighted average between the weekday and weekend day responses. Total television viewing times reported were truncated at 1060 minutes/day. In absence of television viewing guidelines for adults, maternal television viewing time was retained as a continuous variable.

\section{Maternal self-efficacy for limiting children's television viewing at $T 1$ and $T 2$}

Maternal self-efficacy for limiting children's television viewing was assessed using three items from a previously developed scale as no measures to assess self-efficacy in these specific domains were published at the time of assessment [8]. The questions assessed mothers' confidence for saying 'no' to television/DVDs when their child was fussing, providing active play options over television viewing and keeping their baby entertained without using television/ DVDs. These items had good internal reliability in this sample $(\alpha=0.72$ at T1 and $\alpha=0.83$ at T2) and acceptable testretest reliability in a separate sample at child aged 4 months $(0.56-0.77)$ and child aged 19 months $(0.59-0.90)$ old. All questions examining maternal self-efficacy were scored on a 4-point Likert-type scale where $0=$ not at all confident and $4=$ extremely confident. Scores were then generated by taking the average score of the three items.

Data for maternal self-efficacy for limiting children's television viewing was split into tertiles (high, mid, low maternal self-efficacy) at T1 and T2 separately. To assess the direction of change, a categorical variable was created using a similar approach to other tracking studies [24, 25]. This variable consisted of four categories: (1) persistently high self-efficacy (top tertile at T1 and T2 or middle tertile at T1 and T2), (2) persistently low self-efficacy (bottom tertile at T1 and T2), (3) increasing self-efficacy (bottom tertile at $\mathrm{T} 1$ to middle/ top tertile at $\mathrm{T} 2$ or middle tertile at T1 to top tertile at T2) or (4) decreasing self-efficacy (top tertile at $\mathrm{T} 1$ to middle or bottom tertile at T2 or middle tertile at $\mathrm{T} 1$ to bottom tertile at $\mathrm{T} 2$ ).

\section{Children's television viewing time}

At T1 and T2, mothers proxy-reported the total time (hours and minutes) that their child spent watching or in front of the television during the past week. Test-retest reliability of this single item measure from in a separate sample was good $(\mathrm{ICC}=0.84)$. All responses were converted to minutes/week.

\section{Statistical analysis}

Despite no statistical differences in maternal self-efficacy for limiting children's television viewing or tracking categories between intervention and control groups at $\mathrm{T} 1$ or T2, all data were adjusted for intervention group due to exposure to the program. Tracking of maternal self-efficacy for limiting television viewing was assessed using weighted kappa and multinomial logistic regression (reference category: middle tertile at T1 and T2). Linear regression analyses examined associations between maternal self-efficacy tracking categories (persistently high self-efficacy, persistently low self-efficacy, increasing selfefficacy, decreasing self-efficacy) and children's television viewing time at 19-months old, adjusted for intervention group, baseline television viewing time and clustering by first-time mothers group. The odds of being in the different categories of movement based on maternal and child demographic and behavioural predictors were analysed using multinomial logistic regression (reference category: persistently low self-efficacy), controlling for the same covariates described above. All analyses were conducted in Stata 12.0.

\section{Results}

A total of 404 mothers had complete questionnaire data for T1 and T2. Mothers with complete data had children who were slightly younger at $\mathrm{T} 1$ (on average approximately 2 weeks) than those who did not have complete data $(p<0.05)$. No other differences in demographic or behavioural predictors assessed in this study were observed. Demographic characteristics of included participants are outlined in Table 1.

Although significant, the weighted kappa coefficient indicated that tracking of maternal self-efficacy for limiting television viewing was low over the 15 -month time period $(\mathrm{K}=0.23, \mathrm{p}<0.001)$. Compared with mothers who were in the middle tertile at $\mathrm{T} 1$ and $\mathrm{T} 2$, mothers in the top tertile at $\mathrm{T} 1$ were $2.2(95 \% \mathrm{CI}=1.03-4.61)$ times more likely to remain in the top tertile at follow-up. Individuals in the bottom tertile at T1 did not have an increased likelihood of remaining in the bottom tertile at $\mathrm{T} 2$ compared to those in the middle tertile, although the data indicated a trend in this direction $(\mathrm{OR}=1.67 ; 95 \% \mathrm{CI}=0.99-2.80)$. Figure 1 depicts the number of participants within each category of maternal self-efficacy at each time point. The greatest proportion of mothers $(\sim 30 \%)$ had reduced self-efficacy over time, with the lowest proportion of mothers in the persistently high self-efficacy category ( 20\%).

Table 2 reports the associations between the maternal self-efficacy tracking categories and children's television viewing time at 19-months old. Compared to those mothers with persistently low self-efficacy, mothers who had persistently high self-efficacy or increasing self-efficacy had children with lower television viewing behaviour at 
Table 1 Baseline (T1) demographic and behavioural characteristics of participants $(n=404)$

\begin{tabular}{ll}
\hline Child characteristics & T1 \\
\hline Male (\%) & $53.6 \%$ \\
Mean (SD) age (months) & $3.8(1.3)$ \\
Child temperament (\%) & \\
$\quad$ Much easier than average & $15.6 \%$ \\
$\quad$ Easier than average & $40.9 \%$ \\
Average & $35.7 \%$ \\
$\quad$ More/much more difficult than average & $7.7 \%$ \\
Any television viewing time & $58.4 \%$ \\
\hline Mother characteristics & $32.3(4.3)$ \\
\hline Mean age (years) & \\
Maternal education (\%) & $19.6 \%$ \\
Low ( $\leq$ secondary school) & $24.6 \%$ \\
Medium (trade or certificate qualification) & $55.8 \%$ \\
High (university degree +) & $83.9 \%$ \\
Physical activity ( $\geq 150$ mins/week) & $213.6(141.1)$ \\
\hline Mean (SD) television viewing (mins/week)
\end{tabular}

19-months $(\beta=-35.5$ mins; $95 \% \mathrm{CI}=[-54.4,-16.6]$ and $\beta=-37.0 ; 95 \% \mathrm{CI}=[-54.4,-19.7]$, respectively).

Relative to mothers who rated their child's temperament as much easier than average, those who rated their child's temperament as 'average' or 'more difficult than average' had $71 \%$ and $93 \%$ lower odds respectively of having persistently high self-efficacy for limiting television viewing compared to mothers with persistently low self-efficacy for limiting television viewing (Table 3). Additionally, mothers who met the adult physical activity recommendations had 2.5 times greater odds of increasing their self-efficacy for limiting their child's television viewing over time compared to mothers who did not meet physical activity recommendations.

\section{Discussion}

This study was the first to examine tracking of first-time mothers' self-efficacy for limiting television viewing in young children and associations with children's television viewing time. This is important for our understanding of how and when interventions might be best delivered to young families to minimize children's television viewing time. It was found that, although statistically significant, tracking of this construct was low over the 15-month period, with around half of participants moving tertiles between $\mathrm{T} 1$ and $\mathrm{T} 2$, and with a greater proportion of change occurring in a downward direction. This is consistent with cross-sectional work in the field which has found that self-efficacy for limiting television viewing is lower amongst parents of preschool children compared to parents of infants [8]. It is possible that greater movement would be evident if tracking of maternal self-efficacy was assessed over a longer period of time.

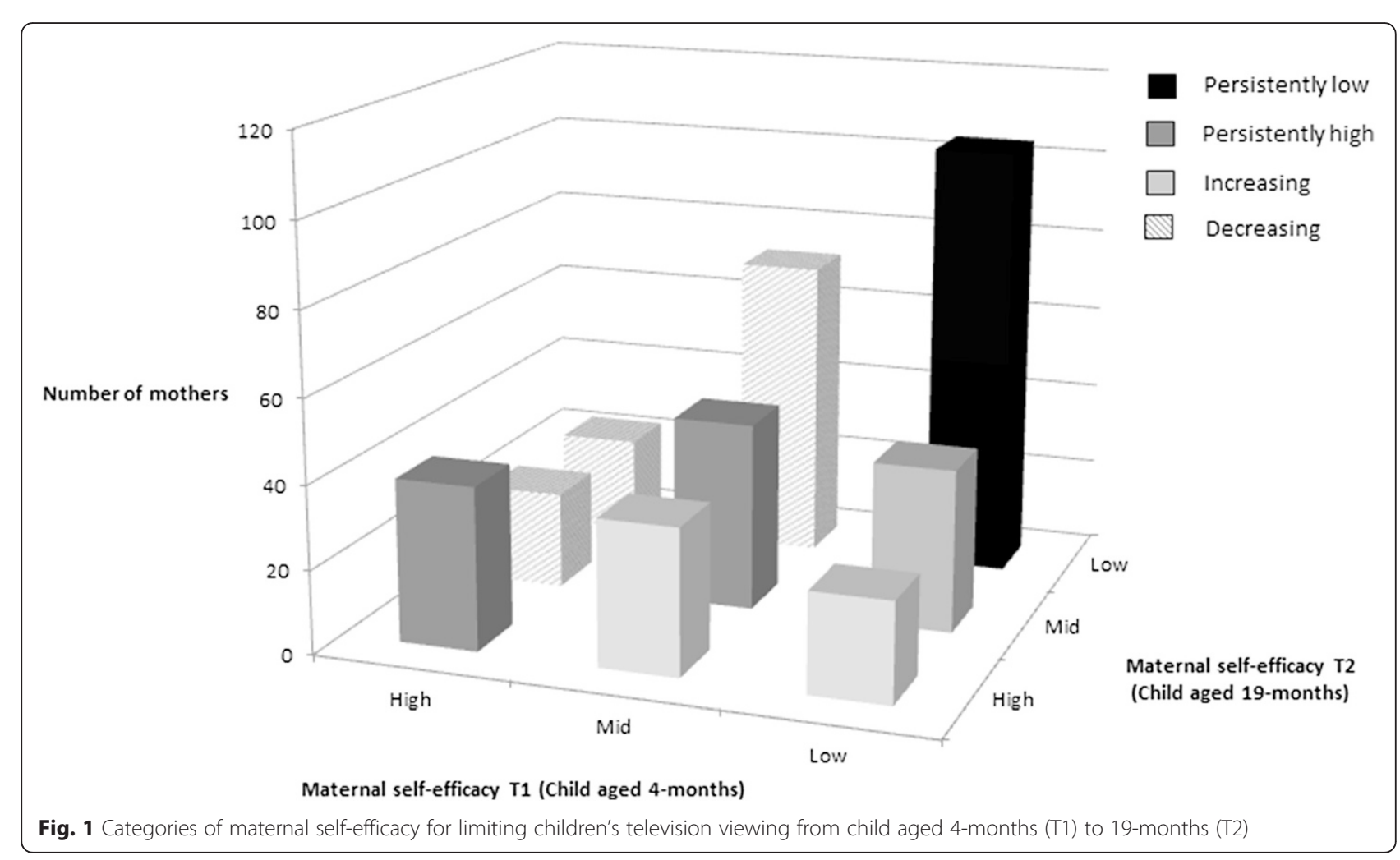


Table 2 Associations between maternal self-efficacy tracking categories and children's television viewing time (mins/week) at 19-months old

\begin{tabular}{ll}
\hline Maternal stability & $\beta(95 \% \mathrm{Cl})$ \\
\hline Persistently low self-efficacy & Ref. \\
Persistently high self-efficacy & $-35.5(-54.4,-16.6)$ \\
Increasing self-efficacy & $-37.0(-54.4,-19.7)$ \\
Decreasing self-efficacy & $-2.6(-22.9,17.7)$
\end{tabular}

Linear regression analyses, adjusted for intervention group, baseline television viewing time and clustering by first-time mothers group

The level of tracking observed in this study varied by tertile rank, with mothers in the extreme tertiles (high or low) at baseline more likely to remain in those tertiles 15-months later. While this is a positive finding for those mothers with high self-efficacy, it is less desirable for mothers with low self-efficacy. Mothers who do not possess particularly high or low self-efficacy at baseline may be more susceptible to changes in selfefficacy, albeit in a positive or negative direction. Thus, an opportunity exists to provide support to these mothers so that the change they may experience is in a favourable direction.

Building from cross-sectional work in the field [7, 8, 11], this study found that favourable changes in maternal self-efficacy over the first year and a half of children's lives are associated with lesser television viewing time, even after controlling for baseline television viewing time. This suggests that supporting mothers to maintain high self-efficacy or intervening to increase maternal self-efficacy over the early childhood period is a worthwhile endeavour to reduce children's television viewing time. In particular, more intensive or targeted support strategies may need to be delivered to mothers who have low self-efficacy for limiting their infant's television viewing from the onset. For example, for mothers with low selfefficacy, providing mothers with alternate activities to help them succeed at limiting television viewing, particularly during challenging situations or child behaviours, may be effective at increasing self-efficacy [10]. For mothers with high self-efficacy, positive reinforcement and feedback may help to maintain their self-efficacy. As interventions to increase parenting self-efficacy in other domains have been successful at improving parenting behaviours and child outcomes [26], developing health promotion programs that focus on enhancing or maintaining maternal self-efficacy for limiting children's television viewing in this early childhood period are critical.

Mothers who rated their child's temperament as 'average' or 'more difficult or much more difficult than average' were less likely to have persistently high self-efficacy for limiting children's television viewing compared to those who rated their child's temperament as 'much easier than average'. The influence of difficult child temperament has previously been investigated in studies around parental feeding practices [27], picky eating [28], weight gain [29] and television viewing [19]. Specific to television viewing, findings by Thompson and colleagues identified that children perceived by their mother to have a more active or fussy temperament were exposed to more television than children with a less active temperament [19]. Drawing from qualitative work [30], it can be hypothesized that in

Table 3 Odds ratios and $95 \%$ confidence intervals $(\mathrm{Cl})^{\mathrm{a}}$ of changing maternal self-efficacy to limit their child's television viewing according to maternal and child predictors among children aged 4- and 19-months ${ }^{\mathrm{b}}$

\begin{tabular}{|c|c|c|c|c|c|c|}
\hline \multirow[t]{2}{*}{ Predictor variable } & \multicolumn{2}{|c|}{ Increasing self-efficacy } & \multicolumn{2}{|c|}{ Decreasing self-efficacy } & \multicolumn{2}{|c|}{ Persistently high self-efficacy } \\
\hline & OR & $95 \% \mathrm{Cl}$ & OR & $95 \% \mathrm{Cl}$ & OR & $95 \% \mathrm{Cl}$ \\
\hline \multicolumn{7}{|l|}{ Maternal physical activity } \\
\hline$<150$ mins/week & 1.0 (ref) & & 1.0 (ref) & & 1.0 (ref) & \\
\hline$\geq 150$ mins/week & $2.49 *$ & $(1.08-5.75)$ & 2.18 & $(0.89-5.32)$ & 1.87 & $(0.90-3.88)$ \\
\hline Maternal TV time & 1.00 & $(0.99-1.01)$ & 1.00 & $(0.99-1.00)$ & 1.00 & $(0.99-1.01)$ \\
\hline \multicolumn{7}{|l|}{ Maternal education } \\
\hline Low & 1.0 (ref) & & 1.0 (ref) & & 1.0 (ref) & \\
\hline Mid & 0.77 & $(0.34-1.76)$ & 1.05 & $(0.50-2.19)$ & 1.45 & $(0.57-3.68)$ \\
\hline High & 0.88 & $(0.36-2.15)$ & 0.99 & $(0.50-1.99)$ & 2.17 & $(0.90-5.27)$ \\
\hline Child age & 0.92 & $(0.71-1.18)$ & 1.10 & $(0.87-1.41)$ & 0.86 & $(0.664-1.15)$ \\
\hline \multicolumn{7}{|l|}{ Child temperament } \\
\hline Much easier than average & 1.0 (ref) & & 1.0 (ref) & & 1.0 (ref) & \\
\hline Easier than average & 0.89 & $(0.34-2.30)$ & 1.01 & $(0.47-2.15)$ & 0.41 & $(0.15-1.10)$ \\
\hline Average & 0.43 & $(0.16-1.14)$ & 0.58 & $(0.24-1.40)$ & $0.29 *$ & $(0.11-0.76)$ \\
\hline More/much more difficult than average & 0.38 & $(0.10-1.47)$ & 0.40 & $(0.12-1.37)$ & $0.07^{*}$ & $(0.01-0.46)$ \\
\hline
\end{tabular}

${ }^{a}$ Multinomial logistic regression, adjusted for intervention group and clustering by first-time mothers group; Reference group: persistently low self-efficacy

${ }^{b}$ Bold results are significant at $\mathrm{p}<0.05$ 
these situations, parents may have opted to use the television to distract the child or calm him or her down or may have been less inclined to restrict television if the child wanted to watch. Supporting this hypothesis is recent findings from Radesky and colleagues [31]. In that study, infants with poor self-regulation (also considered a challenging child behaviour for mothers) at 9-months had higher television viewing time at 2 years-old than their peers with no/mild self-regulation problems.

However, it is also possible that children who exhibit challenging child behaviours (e.g. negative temperament, poor self-regulation) may reduce maternal self-efficacy by impacting on the quality of the mother-child relationship [32]. This negative mother-child relationship may in turn, result in mothers' greater use of television over engagement in interactions with her child. Although there may be many mechanisms to explain the association between child temperament and maternal behaviours, our study highlights that it may be even more critical for mothers of infants with a difficult temperament to have support available (e.g. parenting skills and strategies) from early in their child's life to help them enact alternative strategies to television use to distract, entertain or manage their child.

Although the greatest percentage of mothers decreased tertile ranking in self-efficacy for limiting children's television viewing over time, it is important to note that approximately a quarter of mothers increased their self-efficacy ranking. This may have occurred because mothers were successful at limiting their child's television viewing during the 15-month period and therefore experienced an increase in self-efficacy. Additionally, mothers who met the physical activity recommendations at $\mathrm{T} 1$ were more likely to demonstrate increased self-efficacy. It is possible that more active mothers were more able to provide opportunities for active play or may engage in more physical activity with their children rather than television viewing. It would be beneficial to further investigate mothers who show positive change in self-efficacy over time to identify how and why these changes occur. Such information would be invaluable for the development of future intervention strategies aiming to reduce television viewing in very young children.

This was the first study to assess the tracking of mothers' self-efficacy for limiting her child's television viewing in a relatively large sample; however it was not without limitations. For example, although the time between the two measures of self-efficacy spanned a period of rapid child development (4 months old 19 months old), it is not known whether the pattern of movement would remain consistent over time. In other words, the direction of change in self-efficacy experienced over this relatively short period of time may not be reflective of what parents will experience as their child enters the preschool and primary school years. Additionally, the measures used within this study were all based on maternal self-report and consisted of only a few items as they were part of a larger study. In particular, children's television viewing time was assessed with only one item. More comprehensive assessments and/or the use of objective measures (where possible) may provide less biased estimates. Finally, although this study aimed to capture hypothesized predictors of change in self-efficacy based on previous literature, it is possible that other broader variables not assessed in this study (e.g. maternal depression) may also significantly impact on maternal self-efficacy and children's television viewing time.

It should be noted in the present study that more than half the mothers were highly educated, and many met the physical activity recommendations and engaged in quite low weekly television viewing at baseline compared to the Australian population [23,33]. Thus, these findings may not translate to the general population. Investigating the tracking of maternal self-efficacy in more diverse populations, particularly those with lower levels of education, is desired. Finally, by dividing the data into tertiles (although a common approach for tracking studies $[24,25])$ true stability may have over- or under-estimated if there was substantial movement around or within the cut-off points.

\section{Conclusion}

Findings from the current study suggest support is needed to both increase and maintain maternal self-efficacy for limiting their children's television viewing in the early childhood period, in order to reduce children's later television viewing time. This may be particularly relevant for mothers of children with a difficult temperament. Promoting maternal engagement in physical activity may be a useful strategy for increasing maternal self-efficacy for limiting television viewing. Future research is needed to examine the long-term tracking of maternal self-efficacy and to identify strategies employed by mothers who possess and maintain high self-efficacy for limiting their child's television viewing.

\section{Abbreviations}

MPA: Moderate-intensity physical activity; MVPA: Moderate- to vigorous intensity physical activity; T1: Time point 1; T2: Time point 2; VPA:

Vigorous-intensity physical activity.

\section{Competing interests}

The authors declare that they have no competing interests.

\section{Authors' contributions}

$\mathrm{KC}, \mathrm{KH}$ and JS designed the Melbourne InFANT Program from which the data were drawn. JH analysed the data and drafted the article. KH, JS, KC and NR assisted with data analysis and interpretation, and revised the manuscript for important intellectual content. All authors conceptualized and designed the idea for the paper, agree to be accountable for all aspects of the work and gave approval for the final version to be published. 


\section{Acknowledgements}

Funding for the Melbourne InFANT Program was provided by the National Health and Medical Research Council, Heart Foundation Victoria and Deakin University. JH was supported by a Deakin University International Postgraduate Research Scholarship. JS is supported by a National Health and Medical Research Council Principal Research Fellowship APP1026216. NDR is supported by an Australian Research Council Discovery Early Career Researcher Award (DE120101173). KDH is supported by an Australian Research Council Future Fellowship (FT130100637) and an Honorary Heart Foundation Future Leader Fellowship.

Received: 16 February 2015 Accepted: 21 May 2015

Published online: 30 May 2015

\section{References}

1. American Academy of Pediatrics. Council on Communications Media: Media Use by Children Younger Than 2 Years. Pediatrics 2011, 128(5):1040-1045.

2. LeBlanc AG, Spence JC, Carson V, Gorber SC, Dillman C, Janssen I, et al. Systematic review of sedentary behaviour and health indicators in the early years (aged 0-4 years). Appl Physiol Nutr Metab. 2012;37:753-72.

3. Australian Department of Health and Ageing. National physical activity recommendations for children (0-5 years) [http://www.health.gov.au/ internet/main/publishing.nsf/content/health-pubhlth-strateg-phys-actguidelines]

4. Canadian Society for Exercise Physiology. Canadian sedentary behaviour guidelines for the early years [http://www.csep.ca/english/view.asp?x=804]

5. Zimmerman F, Christakis D, Meltzof A. Television and DVD/video viewing in children younger than 2 years. Arch Pediatr Adolesc Med. 2007;161(5):473-9.

6. Campbell KJ, Crawford DA, Salmon J, Carver A, Garnett SP, Baur LA. Associations between the home food environment and obesity-promoting eating behaviors in adolescence. Obesity. 2007;15(3):719-30.

7. Carson V, Janssen I. Associations between factors within the home setting and screen time among children aged 0-5years: a cross-sectional study. BMC Public Health. 2012;12(1):539.

8. Campbell K, Hesketh K, Silverii A, Abbott G. Maternal self-efficacy regarding children's eating and sedentary behaviours in the early years: Associations with children's food intake and sedentary behaviours. Int J Pediatr Obes. 2010;5(6):501-8.

9. de Silva-Sanigorski A, Ashbolt R, Green J, Calache H, Keith B, Riggs E, et al. Parental self-efficacy and oral health-related knowledge are associated with parent and child oral health behaviors and self-reported oral health status. Community Dent Oral Epidemiol. 2013;41(4):345-52.

10. Bandura A. Self-efficacy: toward a unifying theory of behavioural change. Psychol Rev. 1977;84(2):191-215.

11. Smith B, Grunseit A, Hardy L, King L, Wolfenden L, Milat A. Parental influences on child physical activity and screen viewing time: a population based study. BMC Public Health. 2010;10(1):593.

12. Hesketh K, Hinkley T, Campbell K. Children's physical activity and screen time: qualitative comparison of views of parents of infants and preschool children. Int J Behav Nutr Phys Act. 2012;9(1):152.

13. Malina RM. Tracking of physical activity and physical fitness across the lifespan. Res Q Exerc Sport. 1996;67(3):48-57.

14. Coleman PK, Karraker KH. Parenting self-efficacy among mothers of school-age children: Conceptualization, measurement, and correlates. Fam Relat. 2000:49(1):13-24

15. Leahy-Warren P, McCarthy G. Maternal parental self-efficacy in the postpartum period. Midwifery. 2011;27(6):802-10.

16. Hesketh K, Campbell K, Crawford D, Salmon J, Ball K, McNaughton S, et al. Associations between a mother's own activity levels and her self efficacy and intentions for her child's physical activity. J Sci Med Sport. 2010;12(Supplement 2):e198.

17. Duch H, Fisher E, Ensari I, Harrington A. Screen time use in children under 3 years old: a systematic review of correlates. Int J Behav Nutr Phys Act. 2013;10(1):1-10.

18. Hoyos Cillero I, Jago R. Systematic review of correlates of screen-viewing among young children. Prev Med. 2010;51(1):3-10.

19. Thompson AL, Adair LS, Bentley ME. Maternal characteristics and perception of temperament associated with infant TV exposure. Pediatrics. 2013:131(2):e390-7.
20. Prior M, Sanson A, Smart D, Oberklaid F. Pathways from infancy to adolescence: Australian Temperament Project 1983-2000 (Research Report No. 4). Melbourne: Australian Institute of Family Studies; 2000.

21. Australian Institute of Health and Welfare (AlHW). The Active Australia Survey: a guide and manual for implementation, analysis and reporting. Canberra: AlHW; 2003.

22. Brown WJ, Trost SG, Bauman AE, Mummery K, Owen N. Test-retest reliability of four physical activity measures used in population surveys. J Sci Med Sport. 2004;7(2):205-15.

23. Salmon J, Owen N, Crawford D, Bauman A, Sallis JF. Physical activity and sedentary behavior: A population-based study of barriers, enjoyment, and preference. Health Psychol. 2003;22(2):178-88.

24. Yang X, Telama R, Viikari J, Raitakari O. Risk of obesity in relation to physical activity tracking from youth to adulthood. Med Sci Sports Exerc. 2006;38(5):919-25.

25. Cleland VJ, Ball K, Magnussen C, Dwyer T, Venn A. Socioeconomic position and the tracking of physical activity and cardiorespiratory fitness from childhood to adulthood. Am J Epidemiol. 2009;170(9):1069-77.

26. Jones TL, Prinz RJ. Potential roles of parental self-efficacy in parent and child adjustment: A review. Clin Psychol Rev. 2005;25(3):341-63.

27. McMeekin S, Jansen E, Mallan K, Nicholson J, Magarey A, Daniels L. Associations between infant temperament and early feeding practices. A cross-sectional study of Australian mother-infant dyads from the NOURISH randomised controlled trial. Appetite. 2013:60:239-45.

28. Hafstad GS, Abebe DS, Torgersen L, von Soest T. Picky eating in preschool children: The predictive role of the child's temperament and mother's negative affectivity. Eating Behav. 2013;14(3):274-7.

29. Anzman-Frasca S, Stifter CA, Birch LL. Temperament and childhood obesity risk: a review of the literature. J Dev Behav Pediatr. 2012;33(9):732-45.

30. De Decker E, De Craemer M, De Bourdeaudhuij I, Wijndaele K, Duvinage K, Koletzko B, et al. Influencing factors of screen time in preschool children: an exploration of parents' perceptions through focus groups in six European countries. Obes Rev. 2012:13:75-84

31. Radesky JS, Silverstein M, Zuckerman B, Christakis DA. Infant self-regulation and early childhood media exposure. Pediatrics. 2014;133(5):e1172-8.

32. Scaramella LV, Leve LD. Clarifying parent-child reciprocities during early childhood: The early childhood coercion model. Clin Child Fam Psychol Rev. 2004;7(2):89-107

33. Bauman AE, Ford I, Armstrong T. Trends in population levels of reported physical activity in Australia, 1997, 1999 and 2000. ACT Australia Sports Commission: In. Canberra; 2001

\section{Submit your next manuscript to BioMed Central and take full advantage of:}

- Convenient online submission

- Thorough peer review

- No space constraints or color figure charges

- Immediate publication on acceptance

- Inclusion in PubMed, CAS, Scopus and Google Scholar

- Research which is freely available for redistribution 